\title{
Effectiveness of corifollitropin alfa used for ovarian stimulation of poor responder patients
}

\author{
This article was published in the following Dove Press journal: \\ International Journal of Women's Health \\ 17 October 2016 \\ Number of times this article has been viewed
}

\section{Helmy Selman \\ Leonardo Rinaldi}

One Day Medical Center-IVF Unit, Roma, Italy
Correspondence: Helmy Selman

One Day Medical Center-IVF Unit, Via Attilio Ambrosini I I4, 00 I44 Roma, Italy $\mathrm{Tel}+39645212038$

Email helmy.selman@yahoo.com
Purpose: To evaluate the efficiency and efficacy of corifollitropin alfa (follicle-stimulating hormone-carboxy terminal peptide) in the treatment of poor responder patients.

Methods: A total of 85 poor responder patients with a mean age $40.2 \pm 3.9$ years entered our assisted fertilization program. The patients were prospectively randomized into two groups based on the ovarian stimulation regimen used: group A (study group) $(n=42)$ received clomiphene citrate and corifollitropin alfa for the first 7 days of stimulation followed by recombinant follicle stimulating hormone $(\mathrm{rFSH})$ in a gonadotropin-releasing hormone antagonist protocol, and group B (control group) ( $\mathrm{n}=43$ ) received clomiphene citrate and a daily injection of $\mathrm{rFSH}$ in a gonadotropin-releasing hormone antagonist protocol. We analyzed the stimulation outcome, the number of retrieved oocytes, cleaving embryos, and pregnancy and implantation rates as well. Results: Comparable results were observed between the two groups in terms of demographic data, stimulation outcome, and the number of canceled cycles. There were no differences evident between groups A and B with respect to the number of retrieved oocytes ( $3.0 \pm 0.8$ and $2.7 \pm 0.7$, respectively) and the number of cleaving embryos (1.8 \pm 0.6 and $1.7 \pm 0.7$, respectively). Higher, though not statistically significant, differences were observed in favor of group A compared to group B in terms of pregnancy rate per cycle (19\% and $16.3 \%$, respectively), pregnancy rate per transfer (21.6\% and $17.9 \%$, respectively), and implantation rate (14.7\% and $13.4 \%$, respectively). Also, miscarriage rate was similar between patients treated with corifollitropin alfa and those treated with daily rFSH injection ( $12.5 \%$ and $14.2 \%$, respectively).

Conclusion: The results show that ovarian stimulation with corifollitropin alfa appears to be as efficacious and efficient as daily injection $\mathrm{rFSH}$ regimen to treat patients with poor ovarian response.

Keywords: Long-acting FSH, recombinant FSH, ovarian stimulation, ovarian response, pregnancy

\section{Introduction}

The ovarian stimulation induction is a prerequisite procedure in assisted fertilization technologies. The recruitment of a sufficient number of growing follicles following ovarian stimulation increases the oocyte yield and subsequently the success rate after in vitro fertilization (IVF) treatment. However, some women exhibit a scarce ovarian response following controlled ovarian stimulation, and this phenomenon is mainly observed in the so-called "poor responder" patients. ${ }^{1}$ Poor ovarian response (POR) to gonadotropins affects $\sim 9 \%-24 \%$ of IVF patients, and many other causes could be involved, such as woman's age, endometriosis, genetic disorders, ovarian surgery, or even iatrogenic factors. ${ }^{2,3}$ Until recently, there was insufficient evidence to uniformly define poor responder patients, and the most common criteria used were based on the low number of recovered oocytes following an adequate ovarian stimulation. ${ }^{4-8}$ 
More recently, a first systematic and realistic attempt to identify women who respond poorly to stimulation has been reported by the European Society of Human Reproduction and Embryology. They established and published the so-called "Bologna Criteria", 9,10 which include: 1) advanced women's age ( $>40$ years) or any other risk factor for POR; 2 ) recovering a few number of oocytes ( $<3$ oocytes) following previous ovarian stimulation; and 3) abnormal ovarian reserve test (antral follicle count $<5-7$ or antiMullerian hormone $<0.5-1.1 \mathrm{ng} / \mathrm{mL}$ ). However, it has been established that the presence of two of these criteria after maximal stimulation are sufficient to define a patient as poor responder in the absence of advanced maternal age or abnormal ovarian reserve test. ${ }^{9,10}$

Several ovarian stimulation regimens have been used to treat poor responder women, but the results achieved are still controversial. These protocols include: varying the dose of gonadotropins or the day of menstrual cycle to initiate the stimulation, ${ }^{11,12}$ high dose of gonadotropins in a gonadotropin-releasing hormone (GnRH) long protocol, ${ }^{13,14}$ administration of GnRH and gonadotropins together in the follicular phase (flare-up protocol), ${ }^{15,16}$ pre-cycle estrogen treatment, ${ }^{17}$ growth hormone co-treatment, ${ }^{18}$ gonadotropin suppression with oral contraception before IVF cycle, ${ }^{19}$ using clomiphene citrate for stimulation ${ }^{20}$ or clomiphene citrate with human menopausal gonadotropin in an antagonist GnRH protocol, ${ }^{21}$ and non-stimulated (nature cycle) IVF treatment. ${ }^{22}$ All these stimulation regimens have been used with a limited success, and adequate number and quality of retrieved oocytes in poor responder women still remain the most challenging items in assisted reproductive technologies. ${ }^{23,24}$

A novel recombinant follicle-stimulating hormone ( $\mathrm{rFSH})$ analog, known as corifollitropin alfa, has recently been introduced for ovarian stimulation in patients undergoing IVF treatment. ${ }^{25}$ It contains an alpha subunit, which is identical to that of FSH, coupled to a hybrid subunit of the beta subunit of human FSH and the carboxy-terminal peptide of the beta subunit of human chorionic gonadotropin. ${ }^{26,27}$ Clinical data on corifollitropin alfa have shown that it has longer half-life and slower absorption rate compared to rFSH. ${ }^{28,29}$ Thus, the administration of a single dose of corifollitropin alfa during the early follicular phase of the menstrual cycle is capable of sustaining follicular development for the first week of stimulation unlike rFSH, which must be injected daily. ${ }^{30}$ Moreover, it has been demonstrated that corifollitropin alfa is well tolerated by patients and its use does not present any adverse events or complications. ${ }^{31}$ Because of its bioactivity profile, some authors have suggested that corifollitropin alfa may have a positive effect on stimulation outcome of poor responder women undergoing IVF treatment. ${ }^{32,33}$

In the present study, we evaluated the effect of the long-acting FSH and corifollitropin alfa on stimulation and clinical outcomes, when used for ovarian stimulation of poor responder women.

\section{Methods}

\section{Patient selection}

A total of 85 poor responder women entered our IVF program from July 2013 to December 2015. The patients were considered poor responder according to "Bologna Criteria": 9 1) advanced women's age ( $>40$ years); 2 ) few number of retrieved oocytes ( $<3$ oocytes) following previous ovarian stimulation; and 3) abnormal ovarian reserve test (antral follicle count $<5-7$ or anti-Mullerian hormone $<0.5-1.1 \mathrm{ng} / \mathrm{mL}$ ). The patients were included in the study if they fulfilled all criteria or at least two of them. The patients were randomized before gonadotropin stimulation, using a computer-generated random assignment. Sealed envelopes were used until starting the process of randomization in order to conceal treatment allocation. The patients received all the information regarding the nature of the study and gave their written informed consent. The study was approved by One Day Medical Center ethical committee.

The primary parameters considered for statistical analysis were: the number of recovered oocytes, the number of mature metaphase II oocytes, the number of cleaving embryos, pregnancy rate, and implantation rate. We analyzed also the stimulation outcome, ie, canceled cycles, duration of stimulation, serum estradiol level, endometrial thickness on the day of human chorionic gonadotropin administration, fertilization rate, delivery rate, and miscarriage rate.

\section{Ovarian stimulation}

The patients were prospectively randomized into two groups: corifollitropin (group A), as the study group, and daily rFSH injection (group B) as the control group. In group A, 42 women aged $40.3 \pm 3.9$ years were stimulated with $150 \mathrm{mg} /$ day clomiphene citrate, starting from days 2 to 6 of the cycle. On day 2 of the cycle, a single dose of $150 \mu \mathrm{g}$ of corifollitropin alfa (Elonva, Merck Sharp \& Dohme Limited, Rome, Italy) was administered and thereafter a dose of 225 IU/day of FSH (Gonal F, Merk Serono, Rome, Italy) was administered daily, starting from day 7 of the cycle onward. In group B, 43 women aged $41 \pm 2.6$ years received $150 \mathrm{mg} /$ day clomiphene citrate from days 2 to 5 of the cycle and a dose of $225 \mathrm{IU}$ of $\mathrm{rFSH}$ was administered 
daily, starting from day 3 of the cycle onward. When the leading follicle reached $14 \mathrm{~mm}$, all patients in both groups received $0.25 \mathrm{mg}$ /day $\mathrm{GnRH}$ antagonist. A dose of 10,000 IU of human chorionic gonadotropin (Gonasi HP, IBSA, Lugano, Switzerland) was administered in order to induce the final ovulation maturation and trigger when the leading follicle reached a diameter of $18-20 \mathrm{~mm}$ and at least two follicles of 17-18 mm. Ovum pick-up was performed 36 hours after human chorionic gonadotropin administration and the retrieved oocytes were assessed for their maturity. Mature metaphase II oocytes were inseminated by intra-cytoplasmic sperm injection and the resultant viable embryos were transferred into the patient's uterus. The embryo transfer was performed on day 3 after insemination and a dose of $50 \mathrm{mg}$ /day of progesterone was administered in order to support the luteal phase after embryo transfer.

\section{Statistical analysis}

The data were statistically analyzed using the JMP software (version 4.0.4; SAS Corp., Cary, NC, USA). According to the two-tailed hypothesis, the minimum total sample size required was 84 patients, at least 42 patients per group, in order to reach a statistical power of $80 \%$ with confidence intervals of $95 \%$ and an $\alpha$ level of 0.05 . The two-tailed Student's $t$-test, Fisher's exact test, and two-by-two table were used to analyze the parameters between groups where appropriate, and the significance level was set at $P \leq 0.05$.

\section{Results}

Seventy-six out of the 85 studied women underwent the oocyte retrieval procedure, 37 women in group $\mathrm{A}$ and 39 women in group B. Nine patients were canceled because of ovarian response failure to stimulation: five in group A and four in group B. On comparing between the two groups, no statistically significant differences were observed regarding the primary end points (number of retrieved oocytes, number of cleaving embryos, pregnancy and implantation rates, and all studied secondary end points as well).

As depicted in Table 1, demographic data, stimulation outcome, and cancelation rate were similar in both groups. Table 2 shows comparable embryological results, without any relevant significant differences observed between groups A and B in terms of the number of retrieved oocytes ( $3 \pm 0.8$ and $2.7 \pm 0.7$, respectively), the number of mature oocytes ( $25 \pm 0.8$ and $2.4 \pm 0.8$, respectively), and the number of cleaving transferred ( $1.8 \pm 0.6$ and $1.7 \pm 0.7$, respectively). Higher, though not statistically significant, difference in favor of group A compared to B in terms of pregnancy rate per embryo transfer $(21.6 \%$ in group A and $17.9 \%$ in group B), pregnancy rate per cycle (19\% in group $\mathrm{A}$ and $16.3 \%$ in group B), implantation rate (14.7 in group A and 13.4 in group B), and delivery rate per transfer (13.5 in group A and 10.3 in group B). Ongoing pregnancy rate and miscarriage rate were also similar between the two groups (Table 3).

\section{Discussion}

Ovarian stimulation is an important step in assisted reproduction technologies. It implements the administration of exogenous gonadotropins to induce the development of a number of follicles, thus increasing the oocyte yield and the availability of transferable embryos. In turn, adequate oocyte recruitment and recovery, following ovarian-controlled hyperstimulation, increases the likelihood of conception. However, not all women undergoing IVF treatment respond adequately to gonadotropins' stimulation. This phenomenon occurs mainly in the so-called "poor responder" patients. ${ }^{1}$ Obviously, poor response to gonadotropins' stimulation still remains the most challenging problem in assisted reproduction field. Several ovarian stimulation approaches have been implemented with the aim to improve ovarian response and

Table I Demographic data and stimulation outcome

\begin{tabular}{|c|c|c|c|c|}
\hline Parameters & $\begin{array}{l}\text { Group A } \\
\text { corifollitropin + rFSH }\end{array}$ & $\begin{array}{l}\text { Group B } \\
\text { clomiphene }+ \text { rFSH }\end{array}$ & $\begin{array}{l}\text { Odds ratio } / 95 \% \\
\text { confidence intervals }\end{array}$ & $P$-value \\
\hline No of patients & 42 & 43 & & \\
\hline Mean age $\pm S D$ & $40.3 \pm 3.9$ & $40.9 \pm 2.6$ & $\mathrm{I} .00(0.54-\mathrm{I} .85)$ & 0.88 \\
\hline Mean BMI $\pm S D$ & $25.1 \pm 1.9$ & $24.9 \pm 2.1$ & $\mathrm{I} .03(0.5 \mathrm{I}-2.07)$ & 0.92 \\
\hline No of canceled patients (\%) & $5(\mathrm{I} \mid .9)$ & $4(9.3)$ & $1.27(0.32-5.09)$ & 1.00 \\
\hline No of patients with egg retrieval & 37 & 39 & $0.97(0.52-1.80)$ & 0.92 \\
\hline Duration of stimulation (days) & $13.3 \pm 4.5$ & $12.4 \pm 2.2$ & I.I3 (0.46-2.76) & 1.00 \\
\hline Estradiol level on HCG day (pg/mL) & $\mathrm{I}, 020 \pm 580$ & $995 \pm 658$ & $1.08(0.68-1.70)$ & 0.82 \\
\hline Endometrial thickness on HCG day (mm) & $10.6 \pm 2.4$ & $10.2 \pm 2.1$ & $1.09(0.4 \mid-2.89)$ & 1.00 \\
\hline
\end{tabular}

Note: No statistically significant differences were observed between both groups.

Abbreviations: BMI, body mass index; HCG, human chorionic gonadotropin; $r F S H$, recombinant follicle-stimulating hormone; SD, standard deviation. 
Table 2 Embryological characteristics

\begin{tabular}{|c|c|c|c|c|}
\hline Parameters & $\begin{array}{l}\text { Group A } \\
\text { corifollitropin + rFSH }\end{array}$ & $\begin{array}{l}\text { Group B } \\
\text { clomiphene }+ \text { rFSH }\end{array}$ & $\begin{array}{l}\text { Odds ratio/95\% } \\
\text { confidence intervals }\end{array}$ & P-value \\
\hline No of patients with egg retrieval & 37 & 39 & $0.97(0.52-1.80)$ & 0.92 \\
\hline No of recovered oocytes (mean $\pm \mathrm{SD}$ ) & II $4(3.0 \pm 0.8)$ & $109(2.7 \pm 0.7)$ & $0.96(0.57-1.63)$ & 0.88 \\
\hline No of mature inseminated oocytes (\%) mean \pm SD & $92(80.7)(2.5 \pm 0.8)$ & $94(86.2)(2.4 \pm 0.8)$ & $\mathrm{I} .03(0.6 \mathrm{I}-\mathrm{I} .77)$ & 0.88 \\
\hline No of fertilized $2 \mathrm{PN}$ oocytes (\%) mean $\pm \mathrm{SD}$ & $72(78.2) 1.9 \pm 0.6$ & 74 (78.7) I.8 \pm 0.6 & $1.04(0.59-1.83)$ & 0.86 \\
\hline No of cleaving embryos (\%) mean \pm SD & $66(91.6) 1.8 \pm 0.6$ & $67(90.5) \quad 1.7 \pm 0.7$ & $1.04(0.59-1.83)$ & 0.88 \\
\hline
\end{tabular}

Note: No statistically significant differences were observed between both groups.

Abbreviations: $r F S H$, recombinant follicle-stimulating hormone; SD, standard deviation.

clinical outcome in poor responder women, but the results are still low and are often controversial. ${ }^{11-21}$ The main reason for this controversy is due to the difficulties in establishing a uniform definition of poor responder patients, which can be universally applied. ${ }^{34}$ Recently, the European Society of Human Reproduction and Embryology working group on POR has published a consensus paper describing three criteria to be used to identify women with POR, the so-called "Bologna Criteria". ${ }^{9,10}$ Although these criteria remain the most realistic attempt to identify women with a high probability of having reduced ovarian reserves, further research on the various mechanisms and risk factors, which may differently influence the ovarian reserve, are still urgently needed.

Moreover, a recent Cochrane review, analyzing 10 published trials on different stimulation regimens used for the treatment of poor responder patients, did not find sufficient evidence to indicate any stimulation approach for routine clinical use to treat poor responder women. ${ }^{24} \mathrm{With}$ the advent of DNA technology, a novel recombinant long-acting gonadotropin, corifollitropin alfa, has been recently produced and became available for clinical application. Its efficacy to stimulate multiple follicular growths has been proven in normal responder's women undergoing IVF treatment. ${ }^{28-30}$

A growing body of evidence, as reported in the literature, has widely demonstrated that long-acting FSH (corifollitropin alfa) is noninferior to daily rFSH. A systematic review and meta-analysis included four randomized trials, with a total of 2,326 normal responder patients, on the role of corifollitropin alfa compared to $\mathrm{rFSH}$ in a GnRH antagonist downregulation protocol. The analyzed data showed no significant difference in ongoing pregnancy rate, while an increased risk of developing ovarian hyperstimulation syndrome was observed in patients treated with corifollitropin alfa. ${ }^{35}$ More recently, another meta-analysis, analyzing three randomized controlled trials, involving 3,392 normal responder's women, on corifollitropin alfa compared to $\mathrm{rFSH}$ did not evidence any significant differences in all studied parameters, including pregnancy and live birth rates. The authors concluded that a single dose of corifollitropin alfa for the first 7 days of stimulation is similarly effective as daily injected $\mathrm{rFSH} \cdot{ }^{36}$ Additionally, a Cochrane review including six randomized controlled trials, with a total of 3,753 patients, resulted in no significant differences between patients treated with the long-acting FSH and daily rFSH. From the analyzed data it was concluded that the long-acting FSH is a safe treatment and equally effective compared to daily FSH, but its efficacy in the treatment of hyper or poor responder women is still to be demonstrated. ${ }^{37}$ Moreover, treatment of older women (aged $\geq 35$ to $\leq 42$ years) with corifollitropin alfa has proven to be as efficacious as daily rFSH in terms of the number of recovered oocytes, pregnancy rate, and live birth rate. ${ }^{38}$

Table 3 Clinical outcome

\begin{tabular}{|c|c|c|c|c|}
\hline Parameters & $\begin{array}{l}\text { Group A } \\
\text { corifollitropin }+ \text { rFSH }\end{array}$ & $\begin{array}{l}\text { Group B } \\
\text { clomiphene }+ \text { rFSH }\end{array}$ & $\begin{array}{l}\text { Odds ratio/ } 95 \% \\
\text { confidence intervals }\end{array}$ & $P$-value \\
\hline No of patients who underwent embryo transfer & 37 & 39 & $0.97(0.52-1.80)$ & 0.92 \\
\hline Mean number of embryos transferred \pm SD & $1.8 \pm 0.6$ & $1.7 \pm 0.7$ & $1.04(0.59-1.83)$ & 0.88 \\
\hline Clinical pregnancies per embryo transfer (\%) & $8(21.6)$ & $7(17.9)$ & $0.83(0.27-2.5 \mathrm{I})$ & 0.78 \\
\hline Clinical pregnancies per started cycle (\%) & 19 & 16.3 & $0.85(0.28-2.56)$ & 1.00 \\
\hline Implantation rate $(\%)$ & $9 / 61(14.7)$ & $9 / 67(13.4)$ & $0.91(0.33-2.44)$ & 1.00 \\
\hline Ongoing pregnancies per transfer (\%) & $2(5.4)$ & $2(5.1)$ & $0.94(1.13-7.08)$ & 1.00 \\
\hline Delivery rate per transfer $(\%)$ & $5(13.5)$ & $4(10.3)$ & $0.76(0.19-3.04)$ & 0.73 \\
\hline Miscarriage rate per pregnancy (\%) & I (I2.5) & I (I4.2) & $1.14(0.06-21.87)$ & 1.00 \\
\hline
\end{tabular}

Note: No statistically significant differences were observed between both groups.

Abbreviations: $\mathrm{rFSH}$, recombinant follicle-stimulating hormone; SD, standard deviation. 
Nevertheless, in all preliminary studies on corifollitropin alfa, poor responder patients were excluded from studied population due to their reduced ovarian reserve, ${ }^{37}$ but in our study we used a single dose of corifollitropin alfa administered in the early follicular phase of the menstrual cycle to induce multiple follicular recruitment and growth in poor responder patients undergoing IVF treatment. Previous studies have suggested that corifollitropin alfa might have a valuable role during the early phase of follicular development and growth. Based on the peculiar pharmacokinetic profile and bioactivity of corifollitropin alfa, which is characterized by around two-fold longer half-life in comparison to $\mathrm{rFSH}$, it allows a sustained FSH activity during early follicular recruitment and development. This is a critical step for ovarian stimulation, particularly in the treatment of poor responder women. ${ }^{28,39}$

The use of corifollitropin alfa to treat poor responder patients was first reported by Polyzos et al in two retrospective published studies. ${ }^{32,40}$ In one study, they retrospectively compared poor responder patients stimulated with corifollitropin alfa plus rFSH in a GnRH antagonist regimen, and poor responder patients stimulated with the standard human menopausal gonadotropin in a $\mathrm{GnRH}$ agonist protocol. The obtained results were comparable and the authors concluded that there is no relevant difference between the two groups regarding all studied parameters..$^{32}$ In another retrospective study, the same authors reported on ovarian stimulation of two groups of poor responder women, based on their age: $\leq 40$ years and $\geq 40$ years groups. The patients in both groups were stimulated using corifollitropin alfa plus highly purified human menopausal gonadotropin in a $\mathrm{GnRH}$ antagonist regimen. The author observed a statistically higher pregnancy rate in favor of patients aged $\leq 40$ years compared to those aged $\geq 40$ years ( $28 \%$ and $0 \%$, respectively), whereas all other studied parameters were similar between the two groups. ${ }^{40}$ Recently, a randomized controlled trial showed that there were no statistically significant differences in all studied parameters, including pregnancy and live birth rates, between poor responder patients treated with corifollitropin alfa and those treated with daily rFSH (follitropin beta). ${ }^{41}$ Additionally, Rinaldi and Selman ${ }^{33}$ have retrospectively compared poor responder women treated with corifollitropin alfa plus $\mathrm{rFSH}$ in a $\mathrm{GnRH}$ antagonist regimen and those stimulated with daily rFSH injection in a GnRH antagonist regimen. The achieved results were similar between the two groups for all analyzed parameters, including pregnancy and implantation rates. As the study was analyzed retrospectively and a small number of patients were included, the authors have addressed further studies on the possible positive effect of corifollitropin alfa when used for ovarian stimulation in poor responder patients. ${ }^{33}$

In the present prospective randomized study, we attempt ovarian stimulation in poor responder patients using a single dose of corifollitropin alfa administered in early follicular phase followed by rFSH in comparison to $\mathrm{rFSH}$ daily administered to stimulate poor responder women. In both gonadotropin treatment protocols, clomiphene citrate was added because it has an antiestrogen activity and is capable of interfering with endogenous estrogen in the pituitary gland and hypothalamus by blocking the receptor-binding sites through a feedback-mediating mechanism, resulting in higher production of FSH and LH hormones by the pituitary gland. It also activates the production of a high level of gonadotropins, induces the ovarian follicle's development, maturation, and ovulation. However, although a general agreement on the specific role of clomiphene citrate in ovarian stimulation is still lacking, ${ }^{42}$ some authors have suggested that it may have a positive effect in the treatment of patients with poor ovarian reserve. ${ }^{20,43-45}$

Our results show that corifollitropin alfa exhibits similar results compared with daily injected $\mathrm{rFSH}$ when used to stimulate patients who respond poorly to ovarian stimulation with standard stimulation regimens. Nevertheless, no significant differences were observed between the two groups in terms of all studied parameters, including number of retrieved oocytes, mature oocytes, fertilization rate, pregnancy rate, and implantation rate; evidence of a trend was observed in favor of the patients stimulated with corifollitropin alfa plus $\mathrm{rFSH}$ compared to those treated with daily $\mathrm{rFSH}$.

\section{Conclusion}

Our study shows that corifollitropin alfa appears to be as efficacious and efficient as a daily $\mathrm{rFSH}$ stimulation regimen, and suggests that it can be a reliable alternative stimulation regimen to treat patients with poor ovarian reserve. However, an additional study on a large number of patients should be addressed in order to establish the effective role of the long-acting FSH, corifollitropin alfa, in patients who respond poorly to gonadotropin stimulation.

\section{Author contributions}

All authors contributed toward data analysis, drafting and revising the paper and agree to be accountable for all aspects of the work.

\section{Disclosure}

The authors report no conflicts of interest in this work. 


\section{References}

1. Fauser B, van Heusden A. Manipulation of human ovarian function: Physiological concepts and clinical consequences. Endocr Rev. 1997; 18(1):71-106.

2. Fasouliotis S, Simon A, Laufer N. Evaluation and treatment of low responders in assisted reproductive technology: a challenge to meet. J Assist Reprod Genet. 2000;17(7):357-373.

3. Keay S, Liversedge N, Mathur R, Jenkins J. Assisted conception following poor ovarian response to gonadotrophin stimulation. Br J Obstet Gynaecol. 1997;104(5):521-527.

4. Surrey ES, Schoolcraft WB. Evaluating strategies for improving ovarian response of the poor responder undergoing assisted reproductive techniques. Fertil Steril. 2000;73(4):667-676.

5. Tarlatzis BC, Zepiridis L, Grimbizis G, Bontis J. Clinical management of low ovarian response to stimulation for IVF: a systematic review. Hum Reprod Update. 2003;9:61-76.

6. Bukulmez O, Arici A. Assessment of ovarian reserve. Curr Opin Obstet Gynecol. 2004;16(3):231-237.

7. Kyrou D, Kolibianakis E, Venetis CA, Papanikolaou EG, Bontis J, Tarlatzis BC. How to improve the probability of pregnancy in poor responders undergoing in vitro fertilization: a systematic review and meta-analysis. Fertil Steril. 2009;91(3):749-766.

8. Polyzos NP, Devroey P. A systematic review of randomized trials for the treatment of poor ovarian responders: is there any light at the end of the tunnel? Fertil Steril. 2011;96(5):1058-1061.

9. Ferraretti AP, La Marca A, Fauser BC, Tarlatzis B, Nargund G, Ganaroli L. ESHRE consensus on the definition of 'poor response' to ovarian stimulation for in vitro fertilization: the Bologna criteria. Hum Reprod. 2011;26(7):1616-1624.

10. Ferraretti AP, Gianaroli L. The Bologna criteria for the definition of poor ovrian responders: is there a need for revision. Hum Reprod. 2014;29(9): 1842-1845.

11. Hofmann GE, Toner JP, Muasher SJ, Jones GS. High dose follicle stimulating hormone (FSH) ovarian stimulation in low-responder patients for in vitro fertilization. J Vitro Fertil Embryo Transfer. 1989;6(5): 285-289.

12. van Hoff MH, Alberda AT, Huisman GJ, Zeilmaker GH, Leerentveld RA. Doubling the human menopausal gonadotropin dose in course of an in-vitro fertilization treatment cycle in low responders: a randomized study. Hum Reprod. 1993;8(3):369-373.

13. Serafini P, Stone B, Kerin J, Batzofin J, Quinn P, Marrs RP. An alternate approach to controlled ovarian hyperstimulation in 'poor responders': pretreatment with a gonadotropin-releasing hormone analog. Fertil Steril. 1988;49(1):90-95.

14. Land JA, Yarmolinskaya MI, Dumoulin JC, Evers JL. High-dose human menopausal gonadotropin stimulation in poor responders does not improve in vitro fertilization outcome. Fertil Steril. 1997;65(5): 961-965.

15. Howles CM, Macnamee MC, Edwards RG. Short term use of an LHRH agonist to treat poor responders entering an in-vitro fertilization programme. Hum Reprod. 1987;2(8):655-656.

16. Karande V, Morris R, Rhinehart J, Miller C, Rao R, Gleicher N. Limited success using the 'flare' protocol in poor responders in cycles with low basal follicle-stimulating hormone levels during in-vitro fertilization. Fertil Steril. 1997;67(5):900-903.

17. Russel JB. Pre-cycle estrogen treatment and poor responders. Assist Reprod Rev. 1995;5:82-89.

18. Dor J, Seidman DS, Amudai E, Bider D, Levran D, Mashiach S. Adjuvant growth hormone therapy in poor responders to in-vitro fertilization: a prospective randomized placebo-controlled double-blind study. Hum Reprod. 1995;10(1):40-43.

19. Gonen Y, Jacobsen W, Casper RF. Gonadotropin suppression with oral contraceptives before in-vitro fertilization. Fertil Steril. 1990;53(2): 282-287.

20. Awonuga AO, Nabi A. In vitro fertilization with low-dose clomiphene citrate stimulation in women who respond poorly to superovulation. J Assist Reprod Genet. 1997;14(9):503-507.
21. Abdel Mohsen I, Youssef MAFM, Elashmwi H, Darwish A, Mohsen MN, Khattab SM. Clomiphene citrate plus modified GnRH antagonist protocol for women with poor ovarian response undergoing ICSI treatment cycles: randomized controlled trial. Gynecol Obstet. 2013; $3: 158$.

22. Lindheim SR, Vidali A, Ditkoff E, Sauer MV, Zinger M. Poor responders to ovarian hyperstimulation may benefit from an attempt at naturalcycle oocyte retrieval. J Assist Reprod Genet. 1997;14(3):174-176.

23. Karande V, Gleicher N. A rational approach to the management of low responders in in-vitro fertilization. Hum Reprod. 1999;14(7): 1744-1748.

24. Pandian Z, McTavish AR, Aucott L, Hamilton MP, Bhattacharya S. Interventions for 'poor responders' to controlled ovarian hyper stimulation $(\mathrm{COH})$ in in-vitro fertilisation (IVF). Cochrane Database Syst Rev. 2010;20(1):CD004379.

25. Fauser BC, Alper MM, Ledger W, Schoolcraft WB, Zandvliet A, Mannaerts BM; Engage Investigators. Pharmacokinetics and follicular dynamics of corifollitropin alfa versus recombinant FSH during ovarian stimulation for IVF. Reprod Biomed Online. 2011;22(Suppl 1): S23-S31.

26. Fares FA, Suganuma N, Nishimori K, LaPolt PS, Hsueh AJ, Boime I. Design of a long-acting follitropin agonist by fusing the C-terminal sequence of the chorionic gonadotropin beta subunit to the follitropin beta subunit. Proc Natl Acad Sci U S A. 1992;89(10):4304-4308.

27. Fauser BCJM, Mannaerts BMJL, Devroey P, Leader A, Boime I, Baird DT. Advance in recombinant DNA technology: corifollitropin alfa, a hybrid molecule with sustained follicle-stimulating activity and reduced injection frequency. Hum Reprod Update. 2009;15(3):309-321.

28. Duijkers IJ, Klipping C, Boerrigter PJ, Machielsen CS, De Bie JJ, Voortman G. Single dose pharmacokinetics and effects on follicular growth and serum hormones of a long-acting recombinant FSH preparation (FSH-CTP) in healthy pituitary-suppressed females. Hum Reprod. 2002;17(8):1987-1993.

29. Devroey P, Fauser BC, Platteau P, Beckers NG, Dhont M, Mannaerts BM. Induction of multiple follicular development by a single dose of long-acting recombinant follicle-Stimulating hormone (FSH-CTP, corifollitropin alfa) for controlled ovarian stimulation before in vitro fertilization. J Clin Endocrinol Metab. 2004;89(5):2062-2070.

30. Corifollitropin Alfa Dose-Finding Study Group. A randomized doseresponse trial of a single injection of corifollitropin alfa to sustain multifollicular growth during controlled ovarian stimulation. Hum Reprod. 2008;23(11):2484-2492.

31. Bouloux PM, Handelsman DJ, Jockenhövel F, et al; The FSH-CTP study group. First human exposure to FSH-CTP in hypogonadotrophic hypogonadal males. Hum Reprod. 2001;16(8):1592-1597.

32. Polyzos NP, Devos M, Humaidan P, et al. Corifollitropin alfa followed by rFSH in a GnRH antagonist protocol for poor ovarian responder patients: an observational pilot study. Fertil Steril. 2013;99(2):422-426.

33. Rinaldi L, Selman H. Corifollitropin alfa in poor responders: preliminary results. Austin J In Vitro Fertili. 2014;1(2):3.

34. Shanbhag S, Aucott L, Bhattacharya S, Hamilton MA, McTavish AR. Interventions for 'poor responders' to controlled ovarian hyperstimulation $(\mathrm{COH})$ in in-vitro fertilisation (IVF). Cochrane Database Sys Rev. 2007;24(1):CD004379.

35. Mahmoud Youssef MA, van Wely M, Aboulfoutouh I, El-Khyat W, van der Veen F, Al-Inany H. Is there a place for corifollitropin alfa in IVF/ ICSI cycles? A systematic review and meta-analysis. Fertil Steril. 2012; 97(4):876-885.

36. Griesinger G, Boostanfar R, Gordon K, Gates D, Sisk CMC, Stegmann JB. Corifollitropin alfa versus recombinant follicle-stimulating hormone: an individual patient data meta-analysis. Reprod Biomed Online. 2016;33(1):56-60.

37. Boostanfar R, Shapiro B, Levy M, et al. Large, comparative, randomized double-blind trial confirming noninferiority of pregnancy rates for corifollitropin alfa compared with recombinant follicle-stimulating hormone in a gonadotropin-releasing hormone antagonist controlled ovarian stimulation protocol in older patients undergoing in vitro fertilization. Fertil Steril. 2015;104(1):94-103. 
38. Pouwer AW, Farquhar C, Kremer JA. Long-acting FSH versus daily FSH for women undergoing assisted reproduction. Cochrane Database Syst Rev. 2012;13(6):CD009577.

39. Devroey P, Boostanfar R, Koper NP, Mannaerts BM, Ijzerman-Boon PC, Fauser BC; ENGAGE Investigators. A double-blind, non-inferiority RCT comparing corifollitropin alfa and recombinant FSH during the first seven days of ovarian stimulation using a GnRH antagonist protocol. Hum Reprod. 2009;24(12):3063-3072.

40. Polyzos NP, De Vos M, Corona R, et al. Addition of highly purified HMG after corifollitropin alfa in antagonist-treated poor ovarian responders: a pilot study. Hum Rerod. 2013;28(5):1254-1260.

41. Kolibianakis EM, Venetis CA, Bosdou JK, et al. Corifollitropin alfa compared with follitropin beta in poor responders undergoing ICSI: a randomized controlled trial. Hum Reprod. 2015;30(2):432-440.

42. Ozcan Cenksoy P, Ficicioglu C, Kizilkale O, et al. The comparision of effect of microdose GnRH-a flare-up, GnRH antagonist/aromatase inhibitor letrozole and GnRH antagonist/clomiphene citrate protocols on IVF outcomes in poor responder patients. Gynecol Endocrinol. 2014;30(7):485-489.
43. Mohsen IA, Youssef MAFM, Elashmwi H, Darwish A, Mohsen MN, Khattab SM. Clomiphene citrate plus modified GnRH antagonist protocol for women with poor ovarian response undergoing ICSI treatment cycles: randomized controlled trial. Gynecol Obstet. 2013;3:158: Doi 104172/2161-0932.1000158.

44. Oktem M, Guler I, Erdem M, Erdem A, Bozkurt N, Karabacak O. Comparison of the effectiveness of clomiphene citrate versus letrozole in mild IVF in poor prognosis subfertile women with failed IVF cycles. Int J Fertil Steril. 2015;9(3):286-291.

45. Jovanovic VP, Kort DH, Guarnaccia MM, Sauer MV, Lobo RA. Does the addition of clomiphene citrate or letrazole to gonadotropin treatment enhance the oocyte yield in poor responders undergoing IVF? J Assist Reprod Genet. 2011;28(11):1067-1072.
International Journal of Women's Health

\section{Publish your work in this journal}

The International Journal of Women's Health is an international, peerreviewed open-access journal publishing original research, reports, editorials, reviews and commentaries on all aspects of women's healthcare including gynecology, obstetrics, and breast cancer. The manuscript management system is completely online and includes

\section{Dovepress}

a very quick and fair peer-review system, which is all easy to use. Visit http://www.dovepress.com/testimonials.php to read real quotes from published authors.

\footnotetext{
Submit your manuscript here: http://www.dovepress.com/international-journal-of-womens-health-journal
} 